\title{
Mass-losing AGB stars in Galactic Bulge ISOGAL fields
}

\author{
J.A.D.L. Blommaert
}

ISO Data Centre, Astrophysics Division, Space Science Dept. of ESA, Villafranca, PO Box 50727, E-38080 Madrid, Spain

S. Ganesh*, A. Omont, M. Schultheis

Institut d'Astrophysique de Paris, CNRS, France

*: on leave from Physical Research Laboratory, Ahmedabad, India

D. Ojha

TIFR, Bombay, India

C. Alard, G. Simon

Observatoire de Paris, DASGAL, France

and The ISOGAL Collaboration ${ }^{1,2}$

\begin{abstract}
First results on AGB stars with the ISOGAL mid-infrared survey are presented. We show that M6 and later spectral type giants are detected at 7 and $15 \mu \mathrm{m}$ up to the distance of the Galactic Centre. Analysis of the observed magnitudes and colours indicates that most of the detected AGB stars in the Galactic Bulge are losing mass. ISOCAM CVF spectra on a field in the Bulge confirm the mass loss and also show a large diversity in the shape of the silicate feature.
\end{abstract}

\section{Introduction, the ISOGAL survey}

ISOGAL is a survey at 7 and $15 \mu \mathrm{m}$ of $\approx 22$ degree $^{2}$ of the inner Galaxy. It is performed with ISOCAM (Cesarsky et al. 1996), the mid-infrared imaging device of the Infrared Space Observatory $\left(\mathrm{ISO}^{3}\right)$ satellite. In combination with IJK $_{\mathbf{s}}$ DENIS (Epchtein et al. 1997) data this survey allows studies of cold stellar

\footnotetext{
${ }^{1}$ ISOGAL Co-Investigators include: C. Alard, J. Blommaert, C. Cesarsky, N. Epchtein, M. Felli, P. Fouqué, R. Genzel, G. Gilmore, F. Guglielmo, H. Habing, A. Omont (PI), M. Pérault, S. Price, A. Robin and G. Simon

${ }^{2}$ The ISOGAL data reduction team includes: C. Alard, T. August, J. Blommaert, E. Copet, S. Ganesh, D. Ojha, M. Pérault, M. Schultheis, G. Simon and M. Unavane

${ }^{3}$ ISO, an ESA project with instruments funded by ESA Member States (especially the PI countries: France, Germany, the Netherlands and the United Kingdom) with the participation of ISAS and NASA
} 
populations and galactic structure in regions which are highly obscured in the visible. In comparison with IRAS, the sensitivity and spatial resolution are almost 100 times better. This allows us to study AGB stars in regions which were too confused for IRAS like the galactic plane and the inner Galactic Bulge. Apart from AGB studies, the survey wants to serve several other scientific goals like galactic structure, a characterisation of the small scale interstellar extinction, dusty young stars, diffuse mid-infrared emission. For a further description of ISOGAL see Omont et al. (1998).

ISOGAL will give us a possibility to study large numbers of AGB stars at different positions in the Galaxy and enable us to study dependences of local environment on their evolution (for instance different metallicities). In this contribution we focus on a study of the Bulge and demonstrate the possibilities offered with this new mid-infrared survey.

Systematic cross-identifications with near-infrared I,J, $\mathrm{K}_{\mathbf{s}}$ DENIS sources is performed for all ISOGAL detected sources. It is intended to make a fivewavelength ISOGAL catalogue available by mid-1999 (through CDS and on http://www-isogal.iap.fr/).

\section{Observations and data reduction}

ISOGAL observations were performed throughout the whole lifetime of the ISO satellite (November 1995 - April 1998). Unfortunately a large fraction of the fields were not observed simultaneously at 7 and $15 \mu \mathrm{m}$. The observed regions of $\approx 22$ degree $^{2}$ were distributed along the inner galactic disk (mostly within $\left.|l|<30^{\circ},|b|<1^{\circ}\right)$. The central region $\left(|l|<1.5^{\circ},|b|<0.5^{\circ}\right)$ was almost entirely observed, except very close to the centre itself as that would saturate the detector. For comparison and to extend our study, some fields at larger longitude and at higher latitude (in the Bulge) were observed. A major difficulty for the measurements in the galactic plane was to avoid bright $(\approx 6 \mathrm{Jy}$ at $12 \mu \mathrm{m})$ sources which would saturate the detector. For this reason, the observations were performed in small rasters $\left(\approx 0.1 \mathrm{deg}^{2}\right)$. The pixel field of view selected was $6^{\prime \prime}$ (resulting in a total field of view of $3^{\prime} \times 3^{\prime}$ per raster position). The raster step size was $1.5^{\prime}$. Generally broadband filters were used: LW3 $(12-18 \mu \mathrm{m})$ and LW2 (5-8.5 $\mu \mathrm{m})$ and only short integration times $(40 \times 0.28$ seconds $)$. In special cases, like near the Galactic Centre or on active star formation regions, narrowband filters at the same central wavelengths were used to allow for higher fluxes. The overall sensitivity is better than $10 \mathrm{mJy}$ (corresponding to $10 \mathrm{mag}$. for LW2 and $8.5 \mathrm{mag}$. for LW3). Additionaly, $18\left(3^{\prime} \times 3^{\prime}\right)$ fields were selected for ISOCAM CVF $(5-17 \mu \mathrm{m})$ measurements. One example of this spectroscopic mode will be discussed in section 4 . For a further description of the observations see Omont et al. 1998.

The data reduction consists of several "standard" steps like the removal of cosmic ray impacts, dark current subtraction and flat fielding. Special care is taken for the time-dependent behaviour of the detector, but the corrections for this are still under investigation. In the often crowded fields we use sophisticated source extraction methods. The photometry is done by psf-fitting. For the ISO results we are presenting here, we have a photometric accuracy better than 0.2 mag. (rms). 


\section{AGB stars near the Galactic Centre}

A [15] vs [7]-[15] diagram of sources selected in the direction of the Bulge is shown in Fig. 1. A clear linear sequence of increasing [7]-[15] colour for brighter $15 \mu \mathrm{m}$ fluxes can be seen. The interpretation is that we see here a sequence of increasing mass-losing AGB stars. This hypothesis is investigated by an observation of one particular field (called C32) which is at 1 degree north of the Galactic Centre and which we discuss here further.

The DENIS $K_{s}$ vs J-K $K_{s}$ diagram of the C32 field shows a well-defined redgiant Bulge sequence shifted by a fairly uniform extinction of $A_{v}=6$ mag. Most of the ISOGAL sources have $\left(\mathrm{K}_{\mathrm{s}}\right)_{\mathrm{o}}<8.2 \mathrm{mag}$. (Fig. 2) and are thus above the RGB limit (Tiede et al. 1995). The $\left(K_{s}\right)_{0}$ range of stars also indicates that the spectral type is M5 or M6 and later (Frogel \& Whitford 1987). This is further confirmed in Fig. 2 when comparing with the DENIS data of Miras and Semi-Regulars in the Bulge PG3 field (centered on $(1, b)=(0,-8)$, Schultheis 1998).

Fig. 3 shows again the [15] vs [7]-[15] diagram but now only for the C32 field. The 5 sources with relatively blue colours $([7]-[15]<0.5)$ but high $15 \mu \mathrm{m}$ flux densities ([15] $<6.5$ mag.) were identified through SIMBAD and are most likely foreground stars. In the same figure two theoretical sequences of M-giants with increasing mass-loss rates are overplotted (sequences provided by Martin Groenewegen). The lower line shows the model prediction of a M5 giant (Fluks et al. 1994) with $\mathrm{L}_{*}=2000 \mathrm{~L}_{\odot}$ and a total mass loss rate ranging from $10^{-9}$ to $10^{-7} \mathrm{M}_{\odot} / \mathrm{yr}$. The upper line shows the range for the same mass loss rates but now for a M8 giant of $\mathrm{L}_{*}=2900 \mathrm{~L}_{\odot}$. It clearly follows the observed sequence of points well. In both cases silicate dust (David \& Papoular 1990) with a gas to dust ratio of 200 was used.

The sequence seen in the [15] vs [7]-[15] diagram can thus well be explained in terms of an increasing mass loss rate. The relationship between the (I-J)o colour and the [7]-[15] (Fig. 4) suggests a relationship between later spectral type and higher mass loss rates. It is interesting to point out that at the massloss rates mentioned, there is no sign of an increase of the near-infrared colours. The mass-loss is only noticeable in our mid-infrared data.

Mass loss rates for late $M$ giants have been studied and discussed in papers as early as Reimers (1975) and Gehrz \& Woolf (1971) but it is for the first time that we can observe the onset of the mass loss on the AGB at a distance of 8 kpc. Together with the DENIS data we will be able to get a complete census of the mass loss on the AGB which should give us a better understanding of the importance of the mass loss on the evolution.

It is also interesting to put the number of mass losing stars in the perspective of the variable stars like Miras or Semi-Regulars (SRV), known to undergo mass loss. On the basis of an extrapolation of the number of Miras in the Bulge (Blommaert 1992) we expect to find not more than $20 \mathrm{SRVs}+$ Miras in the C32 field. About 180 ISOGAL sources are known, of which most are in the sequence of mass loss, so much more than the number of Long Period Variables. Interestingly, results of the very sensitive MACHO project (Alves et al. 1998) in the direction of the Magellanic Clouds indicate that $90 \%$ of their discovered red variables (considered to be AGB) have an R-amplitude of less than 0.5 magnitude and would have been undetected in previous LPV surveys. 


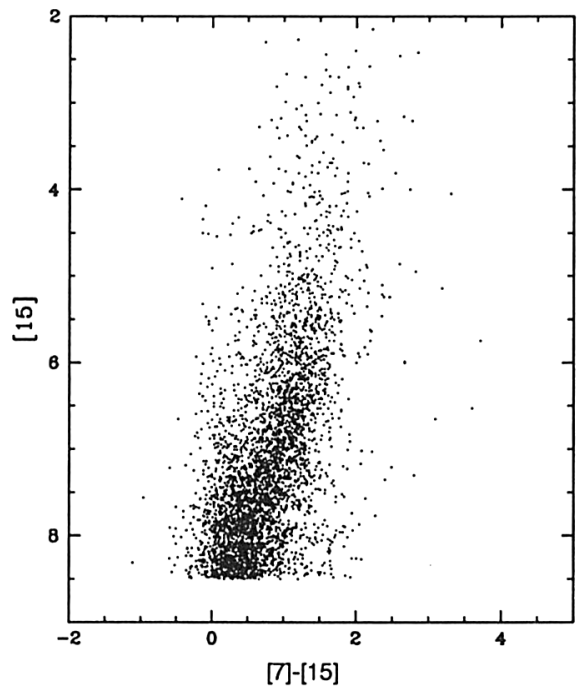

Figure 1. [15] vs [7]-[15] diagram for all ISOGAL fields in the Bulge

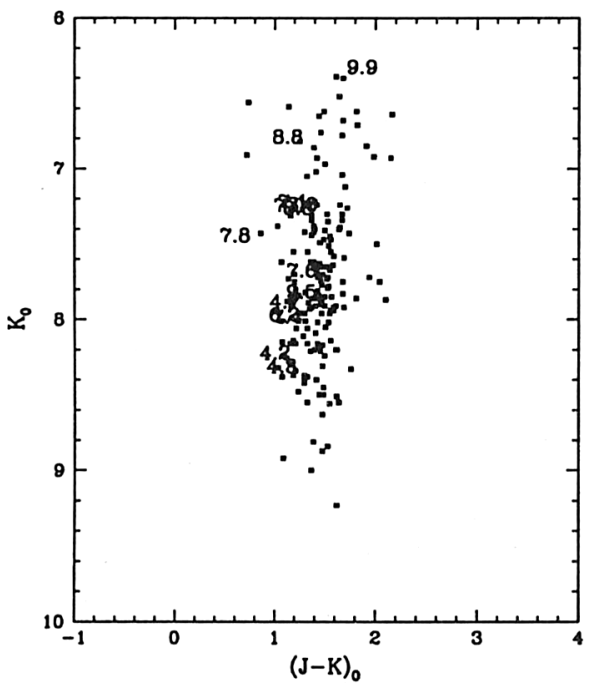

Figure 2. $\left(\mathrm{K}_{\mathrm{s}}\right)_{\mathrm{o}}$ vs $\left(\mathrm{J}-\mathrm{K}_{\mathrm{s}}\right)_{\mathrm{o}}$ diagram of the ISOGAL sources in C32. Overplotted are the spectral types of LPV stars of the PG3 field (shifted by -0.5 in $\left.\left(\mathrm{J}-\mathrm{K}_{\mathrm{s}}\right)_{\mathrm{o}}\right)$.

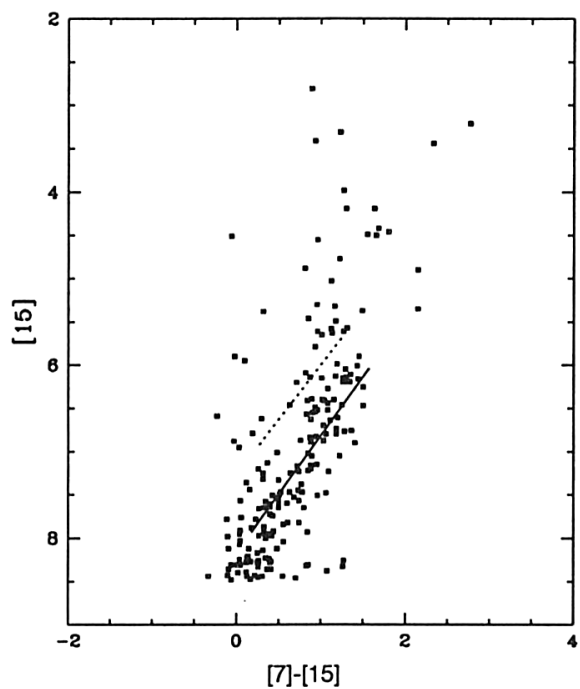

Figure 3. [15] vs [7]-[15] diagram of the C32 field with the theoretical sequences of $M$ giants with increasing mass loss rate.

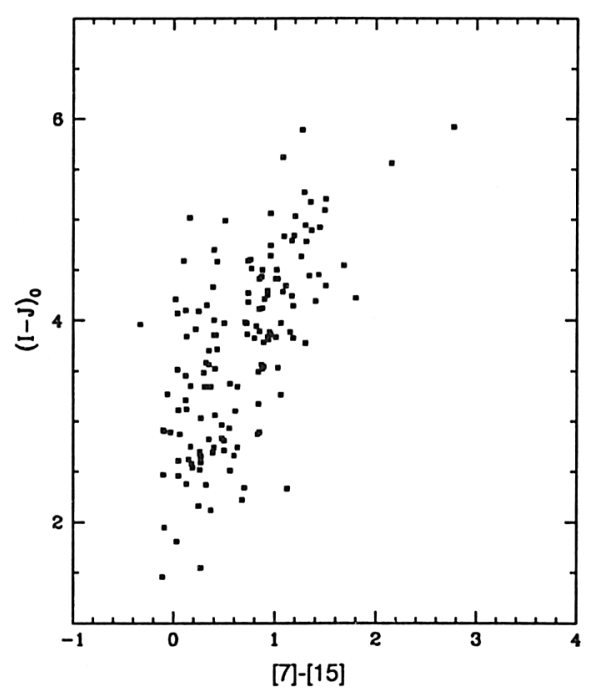

Figure 4. (I-J)。 vs [7]-[15] diagram of the C32 field. 


\section{CVF measurements}

To further substantiate our claim that we see the mass loss of the stars observed in the Bulge we now discuss the $5-15 \mu \mathrm{m}$ spectra taken in an ISOCAM CVF measurement which was performed on a $3^{\prime} \times 3^{\prime}$ subfield of C32. Around 30 objects were detected of which half had a significant flux up to $16 \mu \mathrm{m}$. All these latter sources are also in the catalogue of our ISOCAM filter measurements. In Figs. 5-7 examples are shown of stars in different parts of the [15] vs [7]-[15] diagram. The first object comes from the bottom of the sequence. The dashed line is a M5 giant model (Fluks et al. 1994) shifted to fit the spectrum at $6 \mu \mathrm{m}$. There is no mid-IR excess. One also clearly notices the $\mathrm{SiO}$ band absorption common in late type giants, but not included in the model. The second example shows a source from the middle of the sequence. Here the model of the M5 giant is reasonable well followed up to $\approx 9 \mu \mathrm{m}$ where a silicate feature can be seen, clearly indicative of mass loss. The last example is of a source above the sequence. One further remarkable fact is that we see a very clear diversity of silicate features in our sample.

Acknowledgments. We thank Martin Groenewegen for providing us the model sequences. For the ISOCAM data reduction we used "CIA", a joint development by the ESA Astrophysics Division and the ISOCAM Consortium. The ISOCAM Consortium is led by the ISOCAM PI, C. Cesarsky, Direction des Sciences de la Matière, C.E.A., France.

\section{References}

Alves D., Alcock C., Cook K., et al., 1998, in "Pulsating Stars - Recent Developments in Theory and Observation", XXIII IAU Gen. Assembly, Takenti and Sasselov (eds.), astro-ph/9804003

Blommaert J.A.D.L., 1992, thesis, University of Leiden

Cesarsky C., Abergel A., Agnèse P., et al., 1996, A\&A 315, L32

David P., Papoular R., 1990, A\&A 237, 425

Epchtein N., et al., 1997, The ESO Messenger 87, 27

Fluks M.A., Plez B., Thé P.S., et al., 1994, A\&ASS 105, 311

Frogel J.A., Whitford A.E., 1987, ApJ 320, 199

Gehrz R.D., Woolf N.J., 1971, ApJ 165, 285

Omont A., the ISOGAL Collaboration, 1998, in "Astrophysics with Infrared Surveys: A Prelude to SIRTF", Pasadena, ASP Conference Series, in press

Reimers D., 1975, in "Problems in Stellar Atmospheres and Envelopes", B. Bascheck, W.H. Kegel and G. Traving (eds.), Springer, Berlin, p. 229

Schultheis M., 1998, thesis, University of Vienna

Tiede G.P., Frogel J.A., Terndrup D.M., 1995, AJ 110, 2788 


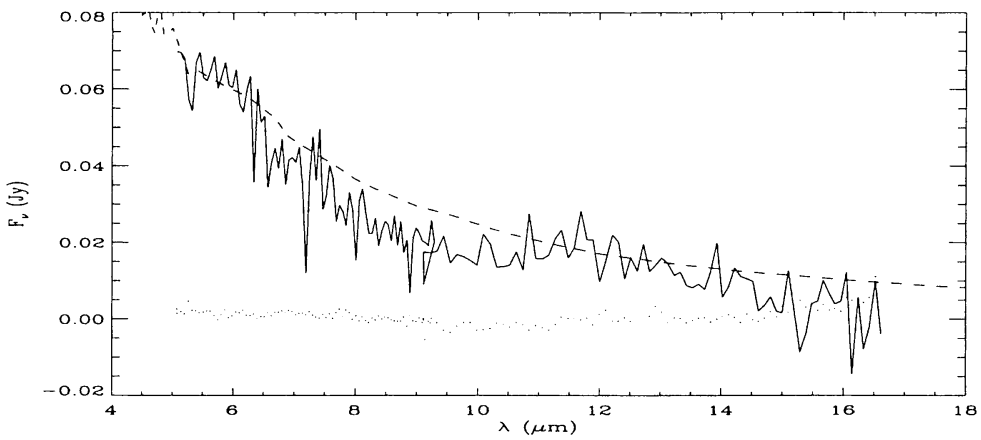

Figure 5. CVF spectrum of a source at the bottom of the sequence seen in the [15] vs [7]-[15] diagram (Fig. 3)

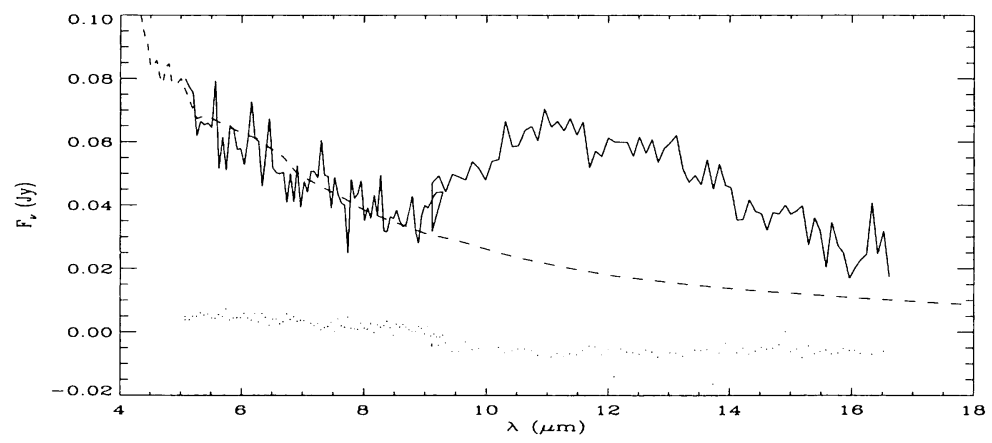

Figure 6. CVF spectrum of a source in the middle of the sequence

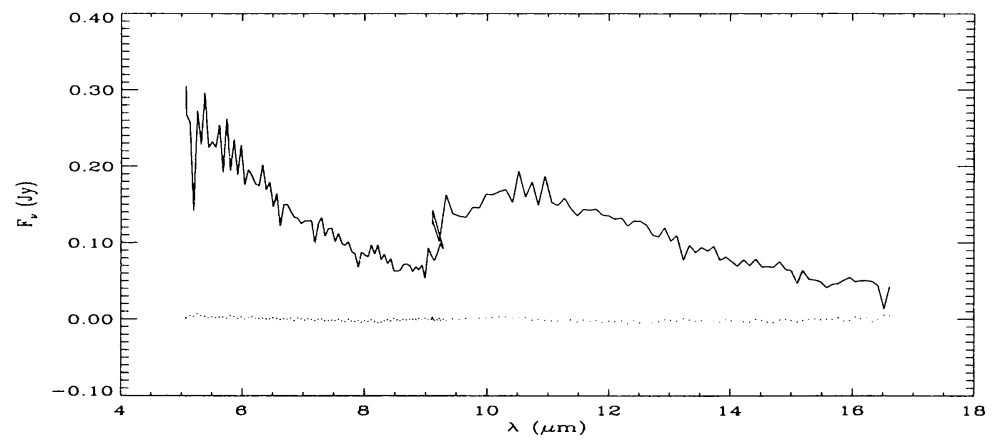

Figure 7. CVF spectrum of a source above the sequence 